\title{
Effect of L-carnitine on health-related quality of life in patients with liver cirrhosis
}

\author{
SHINYA SATO $^{1}$, TADASHI NAMISAKI ${ }^{1}$, MASANORI FURUKAWA ${ }^{1}$, SOICHIRO SAIKAWA ${ }^{1}$, \\ HIDETO KAWARATANI $^{1}$, KOSUKE KAJI ${ }^{1}$, HIROAKI TAKAYA ${ }^{1}$, NAOTAKA SHIMOZATO ${ }^{1}$, \\ YASUHIKO SAWADA $^{1}$, KOH KITAGAWA ${ }^{1}$, KEI MORIYA ${ }^{1}$, TAKEMI AKAHANE ${ }^{1}$, AKIRA MITORO ${ }^{1}$, \\ NORIYUKI HOKI ${ }^{2}$, TATSUICHI ANN ${ }^{2}$ and HITOSHI YOSHIJI ${ }^{1}$ \\ ${ }^{1}$ Department of Gastroenterology and Metabolism, Nara Medical University, Kashihara, Nara 634-8522; \\ ${ }^{2}$ Department of Gastroenterology in Bellland General Hospital, Sakai, Osaka 599-8247, Japan
}

Received March 25, 2020; Accepted July 1, 2020

DOI: 10.3892/br.2020.1372

\begin{abstract}
L-carnitine (4- $N$-trimethylammonium-3hydroxybutyric acid) is the physiologically active form of carnitine and is a natural compound that has been shown to exhibit antioxidant activity. L-carnitine is used as a supplementary treatment in patients with cirrhosis with hepatic encephalopathy, hyperammonemia or muscle cramps. In the present study, the effect of L-carnitine supplementation on health-related quality of life in 30 patients with cirrhosis was prospectively examined. L-carnitine $(1,800 \mathrm{mg} /$ day) was administered orally for 6 months. To assess the effects of L-carnitine on chronic fatigue, patients filled out a self-report questionnaire regarding their physical and mental health. The levels of total and free carnitine, and acylcarnitine were found to be significantly higher 1, 3 and 6 months after therapy initiation compared with before treatment. Serum albumin levels were significantly increased 3 and 6 months after initiation of therapy. L-carnitine supplementation significantly increased the BAP/d-ROM ratio, a marker of antioxidant status in patients with cirrhosis. Changes in serum carnitine concentrations were positively correlated with changes in serum albumin levels $\left(R^{2}=0.369\right.$; $\mathrm{P}=0.012$ ), but not with changes in serum ammonia levels $\left(R^{2}=0.005 ; P=0.78\right)$. Total and mental health scores improved significantly, and physical scores improved marginally 3 and 6 months after initiation of L-carnitine. These findings may be attributed to the enhanced serum albumin levels and oxidative stress rather than the reduced serum ammonia levels. Based on these results, it is suggested that L-carnitine can potentially alleviate chronic fatigue, along with the increased BAP/d-ROM ratio, which were involved in increased oxidative stress in
\end{abstract}

Correspondence to: Dr Tadashi Namisaki, Department of Gastroenterology and Metabolism, Nara Medical University, 840 Shijo-cho, Kashihara, Nara 634-8522, Japan

E-mail: tadashin@naramed-u.ac.jp

Key words: L-carnitine, carnitine profile, albumin, cirrhosis, chronic fatigue, antioxidant activity patients with cirrhosis. The specific mechanisms by which L-carnitine ameliorates chronic fatigue is not fully understood and requires further investigation.

\section{Introduction}

L-carnitine (4- $N$-trimethylammonium-3-hydroxybutyric acid), the biologically active form of carnitine, is synthesized from the essential amino acids, methionine and lysine in certain organs, including the liver (1). Serum L-carnitine concentration varies according to the tissue type $(2)$, nutritional status $(3,4)$ and the quality of the foodstuff (5). L-carnitine regulates the cellular process of generating energy by transferring acyl groups from the cytoplasm to the mitochondrial matrix for $\beta$-oxidation (6). L-carnitine deficiency has been demonstrated to reduce the availability of energy in the liver and is associated with impairments in various aspects of energy metabolism, including utilization and accumulation of carbohydrate and lipids in liver cirrhosis $(7,8)$. Several studies have described the ability of L-carnitine to reduce serum ammonia concentration in clinical trials $(9,10)$. L-carnitine supplementation also reduces the rate of loss of muscle mass (11) and muscle cramps (12) in patients with liver cirrhosis. Intravenous L-carnitine prevents the recurrence of overt hepatic encephalopathy in patients with decompensated cirrhosis (13). Recently, a meta-analysis showed that L-carnitine administration produces favorable effects based on the levels of biochemical markers in patients with cirrhosis (14). Furthermore, its adverse effects are minimal, but may include abdominal pain, nausea, diarrhea and a fishy smell emanating for the patient (15). To the best of our knowledge, there are no studies which have addressed the effect of L-carnitine on health-related quality of life in patients with cirrhosis. The aim of the present study was to evaluate the effects of L-carnitine on chronic fatigue in patients with cirrhosis through the use of self-report questionnaires.

\section{Materials and methods}

Patients. The present study was designed as a clinical trial to prospectively assess the effect of L-carnitine supplementation on chronic fatigue in patients with cirrhosis. The diagnosis of cirrhosis was based on histological examination 


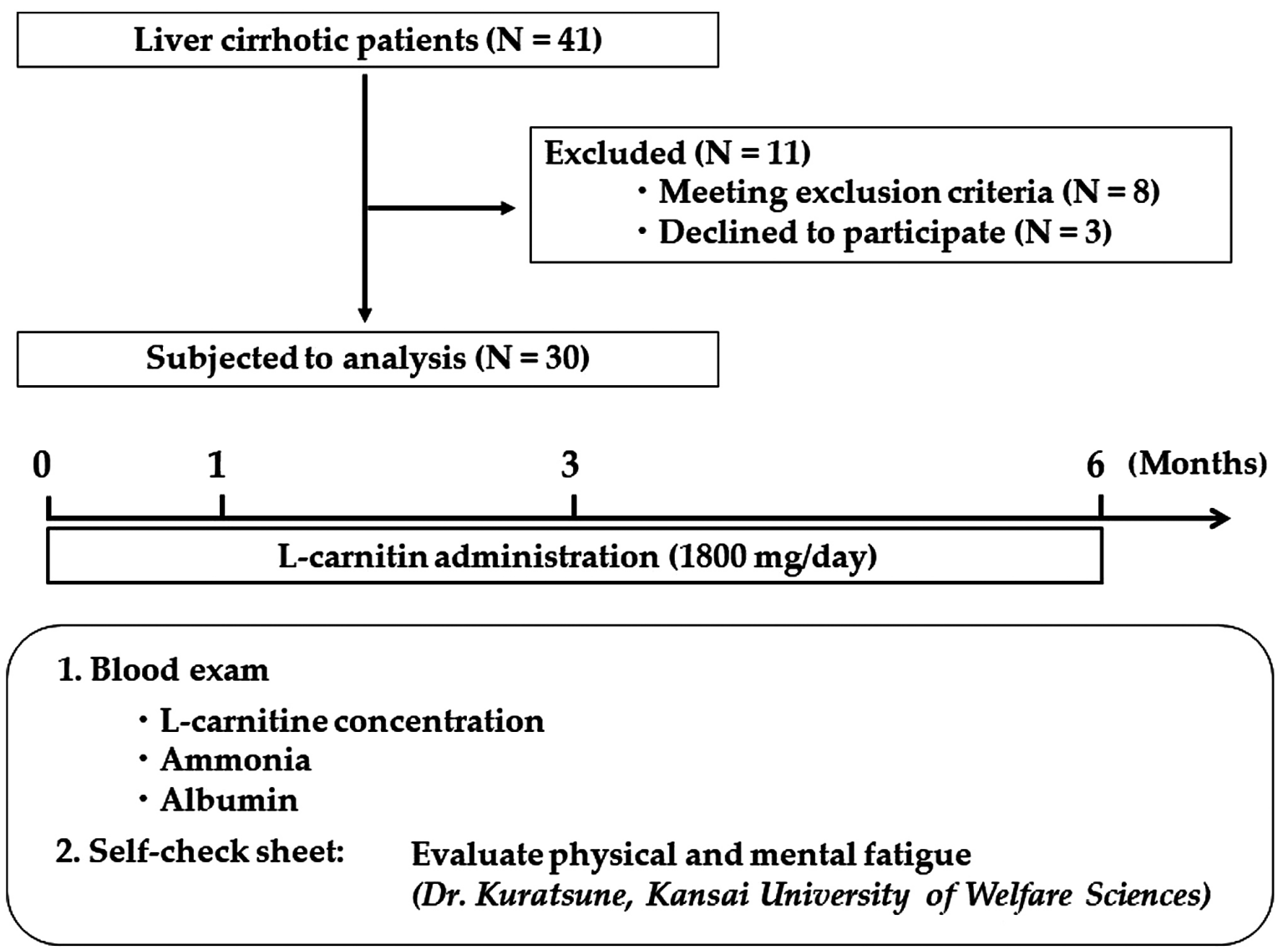

On $0,1,3$, and 6 months

Figure 1. Schematic of the study protocol. Of the 41 prospective patients with cirrhosis, 30 met the inclusion criteria and agreed to participate. Patients were treated for 6 weeks with 1,800 mg of oral L-carnitine per day. Blood samples were tested, and patients filled in the Chronic Fatigue Questionnaire 1, 3 and 6 weeks after treatment initiation.

of biopsy specimens, transient elastography, laboratory tests or imaging findings. Exclusion criteria were evidence of hepatocellular carcinoma, human immunodeficiency virus infection, pregnancy or severe cardiac, pulmonary, or renal diseases. A total of 41 patients with cirrhosis who visited the Department of Gastroenterology in Bellland General Hospital (Osaka, Japan), were asked to participate between April and September 2013 (Fig. 1). Of the 41 patients, 8 patients were excluded as they met at least one of the exclusion criteria, and 3 patients refused to participate; thus, a total of 30 patients were enrolled in the present study. The recruited cohort consisted of 30 patients (median age 69 years; age range, $43-78$ years) with $19(63.3 \%)$ men and 11 (36.7\%) women. Patients took $600 \mathrm{mg}$ (as two $300 \mathrm{mg}$ tablets) of levocarnitine chloride (L-carnitine; Otsuka Pharmaceutical Co., Ltd.) three times a day (total daily dose, 1,800 mg) for 6 months. Blood samples were collected before treatment and 1, 3 and 6 months after L-carnitine supplementation was initiated, to measure the concentrations of several biochemical parameters, free and total carnitine, and acylcarnitine levels. Multiple factors affect the accuracy of ammonia levels. The patients were told not to exercise or smoke cigarettes $12 \mathrm{~h}$ before the fasting blood test was performed. The level of fasting venous ammonia was measured. The blood samples were immediately placed on ice after collection and was centrifuged within 15 min of collection, as described previously (16). None of the patients recruited in the final cohort were prescribed either cyanide, carbamazepine, valproic acid or iron, which can cause secondary hyperammonemia as a result of disruption of mitochondrial pathways $(17,18)$. The initial version of Child-Pugh score, included two continuous variables (bilirubin and albumin) and three discrete (quantitative) variables (ascites, encephalopathy and nutritional status) (19). The selection of these five variables, as well as the cut-off values for bilirubin and albumin were empirical. The five variables and their respective cut-off values were arranged so as to define three distinct groups of increasing severity (A, B and C). Model For End-Stage Liver Disease (MELD) scores had been originally created with the aim of predicting survival after transjugular intrahepatic portosystemic shunt. The score (the ancestor of the current MELD score), derived from a survival function, was calculated as follows: $R=0.957$ $\log _{\mathrm{e}}($ creatinine $[\mathrm{mg} / \mathrm{dl}])+0.378 \log _{\mathrm{e}}($ bilirubin $[\mathrm{mg} / \mathrm{dl}])+1.120$ loge (Prothrombin Time-International Normalized Ratio)+0.643 (cause of cirrhosis). Before and after L-carnitine supplementation, patients were asked to complete the Chronic Fatigue Questionnaire (20). The severity of fatigue was reflected in self-reported ratings, based on daily activities and was represented by the performance status score $(21,22)$. Informed consent was obtained from all participants before the initiation of the study. The present study was approved by the Human Ethics Review Committee of Bellland General Hospital (approval no. 2015-0004) and was performed in 
accordance with the Declaration of Helsinki (23). Subjects were monitored for safety and tolerance of L-carnitine during the course of the study.

Measurement of oxidative stress markers [d-reactive oxygen metabolites (ROMs)] and antioxidant capacity [biological antioxidant potential (BAP)]. Measurement of oxidative stress markers was performed by measuring oxidative stress in vivo with simple and accurate tests, d-ROMs-derived compounds and BAP test. The collected samples were immediately centrifuged at $3,000 \times \mathrm{g}$ at $4^{\circ} \mathrm{C}$ for $10 \mathrm{~min}$ to separate the serum. The separated samples were stored in a freezer at $-20^{\circ} \mathrm{C}$ until further use. Before the assay was initiated, serum levels of d-ROMs and BAP were measured using commercial kits (Diacron International: Cat. nos. AT3274 and BR4873, respectively) on an Olympus AU640 (Olympus, Japan) according to manufacturer's protocol (24). The results of the d-ROMs analysis are expressed in Carratelli units (U.Carr), where 1 U.Carr corresponds to $0.8 \mathrm{mg} / 1 \mathrm{H}_{2} \mathrm{O}_{2}$. The results of the BAP analysis are expressed in $\mu \mathrm{mol} / 1$ of the reduced ferric ions $(25,26)$. Antioxidant/oxidant (BAP/dROMs) ratio was calculated to express relative antioxidant capacity.

Chronic Fatigue Questionnaire. Patients' health status was quantified using the Chronic Fatigue Questionnaire at four different time points: Before treatment, and 1, 3 and 6 months after the initiation of treatment. All patients enrolled in the present study completed the Chronic Fatigue Questionnaire, a 20-item_self-report questionnaire used to examine physical and mental functioning, originally produced by Kuratsune et al (27) and Mizuno et al (28). All items were scored from 0-4; the physical health subscale and the mental health subscale each had 10 items. The physical and mental health scores reflected the mental and physical fitness performance, respectively. To calculate the total score, the scores of each item were added; thus the total score ranged from 0-40 points, with higher scores indicating greater fatigue and/or greater effects of fatigue on quality of life.

Statistical analysis. Numerical variables were calculated as the mean \pm standard deviations. Quantitative parameters were compared using a one-way ANOVA with a post-hoc Tukey's tests. Spearman's rank correlation analysis was used to determine correlations. $\mathrm{P}<0.05$ was considered to indicate a statistically significant difference. All statistical analyses were performed using SPSS version 20.0 (IBM, Corp.).

\section{Results}

Patient characteristics. The characteristics of the patients are listed in Table I. The recruited cohort consisted of 30 patients. The cause of liver disease was hepatitis $C$ viral infection in 14 patients, hepatitis $\mathrm{B}$ viral infection in 2 patients, alcohol abuse in 9 patients, and other disorders, including primary biliary cholangitis, autoimmune hepatitis and idiopathic causes in 5 patients. The number of patients with Child-Pugh classifications (19) of A, B and C were 18, 10 and 2, respectively. The median serum level of ammonia was $91.0 \pm 52.0 \mu \mathrm{g} / \mathrm{dl}$, and that of albumin was $3.3 \pm 0.5 \mathrm{~g} / \mathrm{dl}$. No adverse reactions attributable to L-carnitine administration were observed.
Table I. Clinical and demographic characteristics of patients with cirrhosis.

\begin{tabular}{lc}
\hline Characteristics & Value $^{\mathrm{a}}$ \\
\hline Age, years & $71.0 \pm 8.4$ \\
Sex, male/female & $19 / 11$ \\
Etiology, HBV/HCV/Alcohol/Others & $2 / 14 / 9 / 5$ \\
Child-Pugh classification, A/B/C & $10 / 18 / 2$ \\
Aspartate transaminase, IU/l & $39.5 \pm 21.1$ \\
Alanine aminotransferase, IU/l & $40.2 \pm 18.5$ \\
Alkaline phosphatase, IU/l & $230 \pm 102$ \\
$\gamma$-glutamyl transpeptidase, U/1 & $31 \pm 30$ \\
Total bilirubin, mg/dl & $1.5 \pm 0.8$ \\
Blood urea nitrogen, mg/dl & $13 \pm 10$ \\
Total cholesterol, mg/dl & $165 \pm 39$ \\
Triglyceride, $\mathrm{mg} / \mathrm{dl}$ & $90 \pm 52$ \\
Albumin, $\mathrm{g} / \mathrm{dl}$ & $3.3 \pm 0.5$ \\
Platelets, $\mathrm{x} 10^{4} / \mu \mathrm{l}$ & $11.4 \pm 5.5$ \\
Prothrombin time, $\%$ & $71.9 \pm 15.1$ \\
Ammonia, $\mu \mathrm{g} / \mathrm{dl}$ & $91 \pm 52$ \\
Total carnitine, $\mu \mathrm{mol} / \mathrm{l}$ & $66.5 \pm 12.8$ \\
Free carnitine, $\mu \mathrm{mol} / \mathrm{l}$ & $52.4 \pm 12.1$ \\
Acylcarnitine, $\mu \mathrm{mol} / \mathrm{l}$ & $14.1 \pm 3.7$ \\
& \\
\hline
\end{tabular}

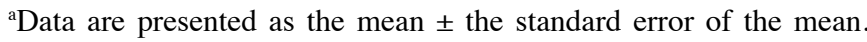
$\mathrm{HBV}$, hepatitis B virus; $\mathrm{HCV}$, hepatitis $\mathrm{C}$ virus.

Effects of 6-month of L-carnitine supplementation on serum levels of total carnitine, free carnitine and acylcarnitine. The levels of total carnitine, free carnitine and acylcarnitine were significantly higher 1, 3 and 6 months after therapy initiation compared with before treatment was initiated. The mean serum levels of total carnitine were $66.1 \pm 10.5 \mu \mathrm{mol} / 1$ before treatment, $108.2 \pm 25.2 \mu \mathrm{mol} / 1$ after 1 month of treatment $(\mathrm{P}<0.05), 122.0 \pm 21.2 \mu \mathrm{mol} / 1$ after 3 months $(\mathrm{P}<0.05)$ and $117.2 \pm 12.2 \mu \mathrm{mol} / 1$ after 6 months $(\mathrm{P}<0.05$; Fig. $2 \mathrm{~A})$. The mean serum levels of free carnitine were $52.4 \pm 9.1 \mu \mathrm{mol} / 1$ before treatment, $83.8 \pm 15.3 \mu \mathrm{mol} / 1$ after 1 month $(\mathrm{P}<0.05)$, 93.2 $\pm 17.1 \mu \mathrm{mol} / \mathrm{l}$ after 3 months $(\mathrm{P}<0.05)$ and $87.5 \pm 16.2 \mu \mathrm{mol} / 1$ after 6 months $(\mathrm{P}<0.05$; Fig. 2B). Mean serum levels of acylcarnitine were $14.1 \pm 3.1 \mu \mathrm{mol} / 1$ before treatment, $24.5 \pm 10.5 \mu \mathrm{mol} / 1$ after 1 month $(\mathrm{P}<0.05), 29.1 \pm 8.3 \mu \mathrm{mol} / 1$ after 3 months $(\mathrm{P}<0.05)$ and $29.4 \pm 8.5 \mu \mathrm{mol} / 1$ after 6 months $(\mathrm{P}<0.05$; Fig. 2C).

Effects of 6-month L-carnitine supplementation on serum levels of albumin and ammonia. Serum albumin levels did not change significantly 1 month after L-carnitine therapy was initiated, but they were significantly higher after 3 and 6 months of supplementation compared with before treatment initiation. Mean serum levels were $3.2 \pm 0.5 \mu \mathrm{g} / \mathrm{dl}$ before treatment, 3.3 $\pm 0.4 \mu \mathrm{g} / \mathrm{dl}$ after 1 month $(\mathrm{P}=0.78), 3.4 \pm 0.3 \mu \mathrm{g} / \mathrm{dl}$ after 3 months $(\mathrm{P}<0.05)$ and 3.6 $\pm 0.3 \mu \mathrm{g} / \mathrm{dl}$ after 6 months $(\mathrm{P}<0.05$; Fig. 3A). In all patients, serum ammonia levels did not change significantly 1 month after L-carnitine therapy was initiated, but they were significantly higher 3 and 6 months after therapy 


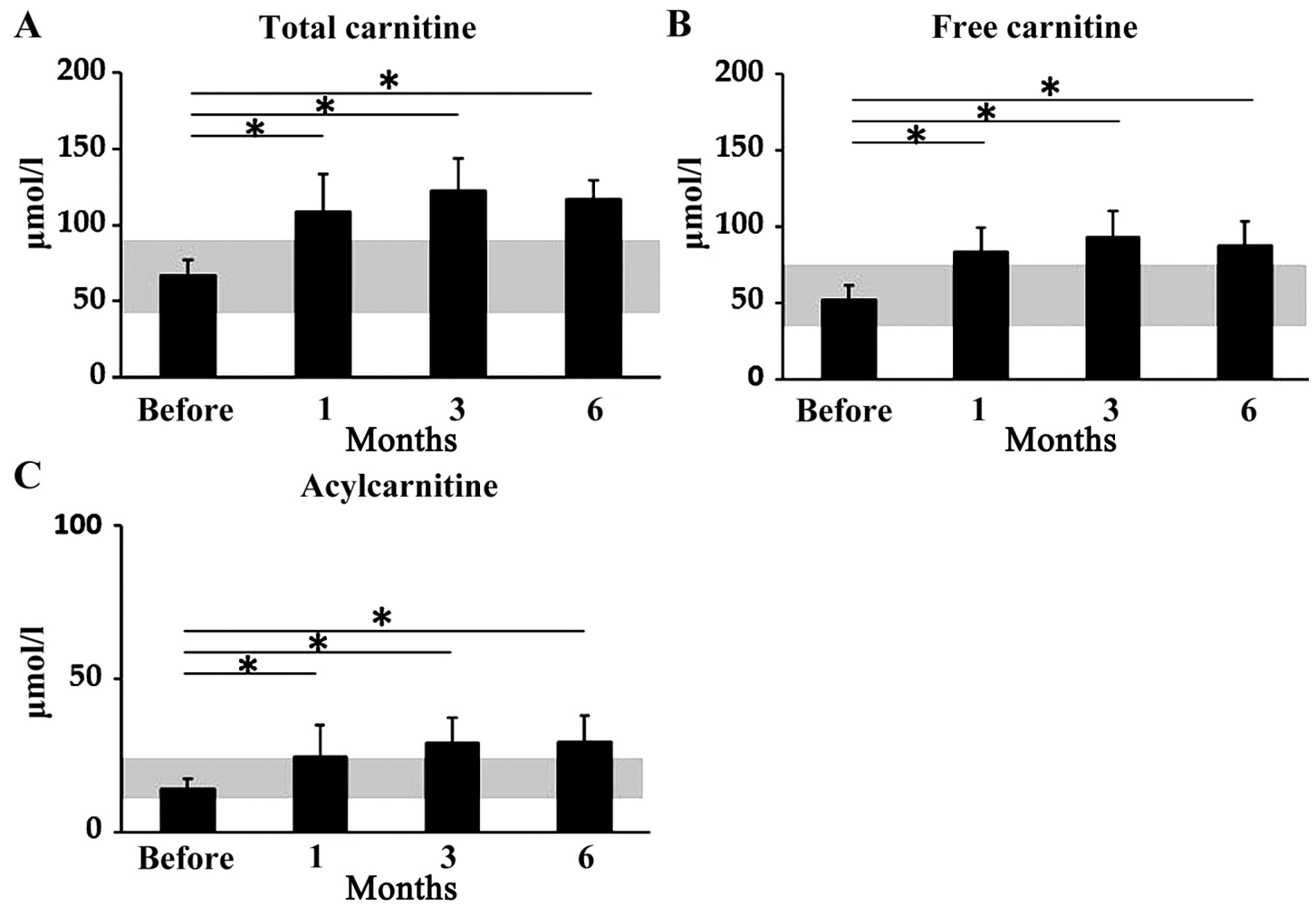

Figure 2. Changes in carnitine profiles during L-carnitine supplementation in patients with cirrhosis. After 1, 3 and 6 months of L-carnitine treatment, levels of (A) total carnitine, (B) free carnitine and (C) acylcarnitine were significantly higher compared with before treatment. Total carnitine levels were $66.1 \pm 10.5 \mu \mathrm{mol} / 1$ before treatment, $108.2 \pm 25.2 \mu \mathrm{mol} / 1$ after 1 month, $122.0 \pm 21.2 \mu \mathrm{mol} / 1$ after 3 months and $117.2 \pm 12.2 \mu \mathrm{mol} / 1$ after 6 months. Free carnitine levels were $52.4 \pm 9.1 \mu \mathrm{mol} / 1$ before treatment, $83.8 \pm 15.3 \mu \mathrm{mol} / 1$ after 1 month, $93.1 \pm 17.1 \mu \mathrm{mol} / 1$ after 3 months and $87.5 \pm 16.2 \mu \mathrm{mol} / 1$ after $6 \mathrm{months}$. Acylcarnitine levels were $14.1 \pm 3.1 \mu \mathrm{mol} / 1$ before treatment, $24.5 \pm 10.5 \mu \mathrm{mol} / 1$ after 1 month, $29.2 \pm 8.3 \mu \mathrm{mol} / 1$ after 3 months and $29.4 \pm 8.5 \mu \mathrm{mol} / 1$ after 6 months. The gray area indicates the physiological range. ${ }^{*} \mathrm{P}<0.05$.

A

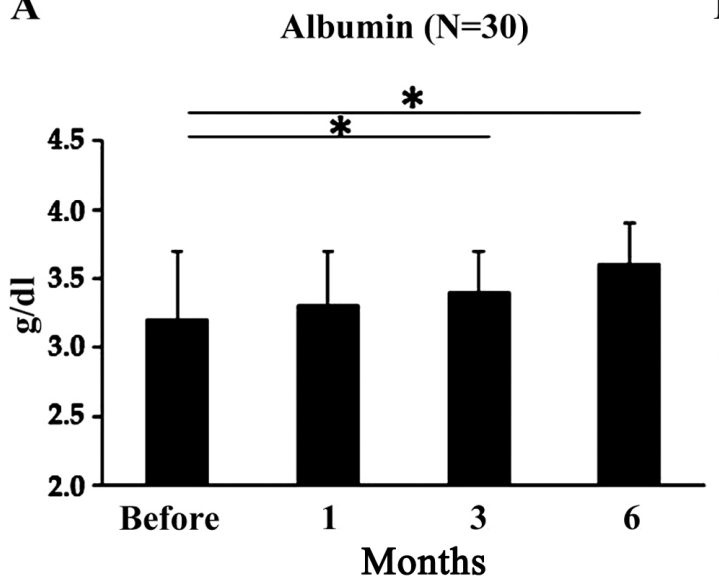

B

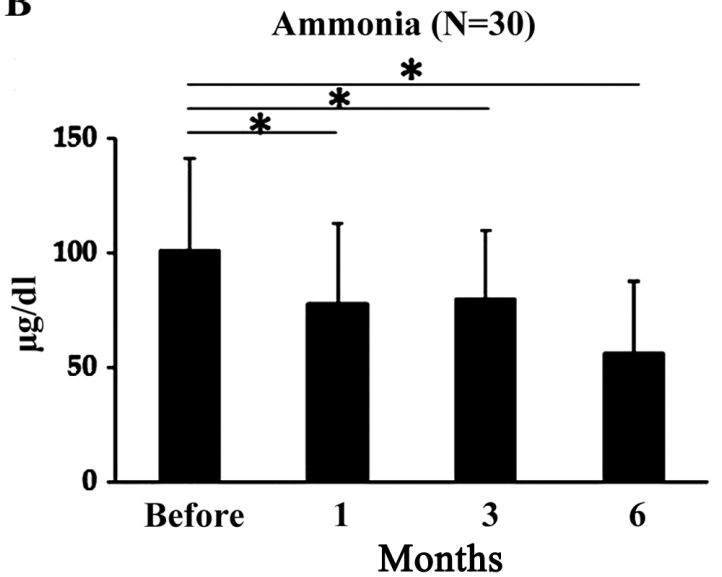

Figure 3. Changes in serum albumin and ammonia levels during L-carnitine supplementation in patients with cirrhosis. (A) L-carnitine supplementation for 3 and 6 months significantly increased serum albumin levels: $3.4 \pm 0.4 \mu \mathrm{g} / \mathrm{dl}$ ( 3 months) and $3.4 \pm 0.3 \mu \mathrm{g} / \mathrm{dl}$ ( 6 months). In contrast, there was no significant effect of L-carnitine supplementation after 1 month on serum albumin levels: $3.2 \pm 0.5 \mu \mathrm{g} / \mathrm{dl}$ before treatment and $3.3 \pm 0.4 \mu \mathrm{g} / \mathrm{dl}$ after $1 \mathrm{month}(\mathrm{P}=0.78)$. (B) Ammonia levels were significantly lower after 6 months of L-carnitine supplementation $(56.5 \pm 31 \mu \mathrm{g} / \mathrm{dl})$ compared with before treatment (101 $\pm 40 \mu \mathrm{g} / \mathrm{dl})$, and they showed a trend toward decreasing after 1 month $(78 \pm 35 \mu \mathrm{g} / \mathrm{dl} ; \mathrm{P}=0.073)$ and 3 months $(80 \pm 30 \mu \mathrm{g} / \mathrm{dl} ; \mathrm{P}=0.074)$ of therapy. $\mathrm{P}<0.05$.

initiation compared with before treatment initiation. Mean serum ammonia levels were $101 \pm 40 \mu \mathrm{g} / \mathrm{dl}$ before treatment, $78 \pm 35 \mu \mathrm{g} / \mathrm{dl}$ after 1 month $(\mathrm{P}<0.05), 80 \pm 30 \mu \mathrm{g} / \mathrm{dl}$ after 3 months $(\mathrm{P}<0.05)$ and $56.5 \pm 31 \mu \mathrm{g} / \mathrm{dl}$ after 6 months $(\mathrm{P}<0.05$; Fig. 3B $)$.

Effects of 6-month L-carnitine supplementation on Child-Pugh and MELD scores. L-carnitine supplementation did not have any significant effect on Child-Pugh or MELD scores. The Child-Pugh score was $7.3 \pm 1.4$ before treatment, $7.2 \pm 1.5$ after 1 month $(\mathrm{P}=0.58), 7.2 \pm 1.6$ after 3 months $(\mathrm{P}=0.38)$ and $7.2 \pm 1.9$ after 6 months $(\mathrm{P}=0.32$; Fig. $4 \mathrm{~A})$. The MELD score was $6.6 \pm 0.9$ before treatment, $6.7 \pm 0.9$ after 1 month $(\mathrm{P}=0.32), 6.6 \pm 1.1$ after 3 months $(\mathrm{P}=0.74)$ and $6.8 \pm 1.5$ after 6 months $(\mathrm{P}=0.65$; Fig. 4B). 
A

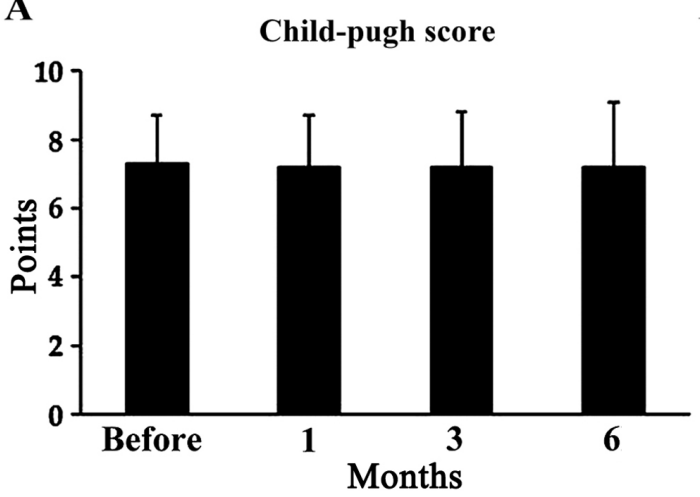

B

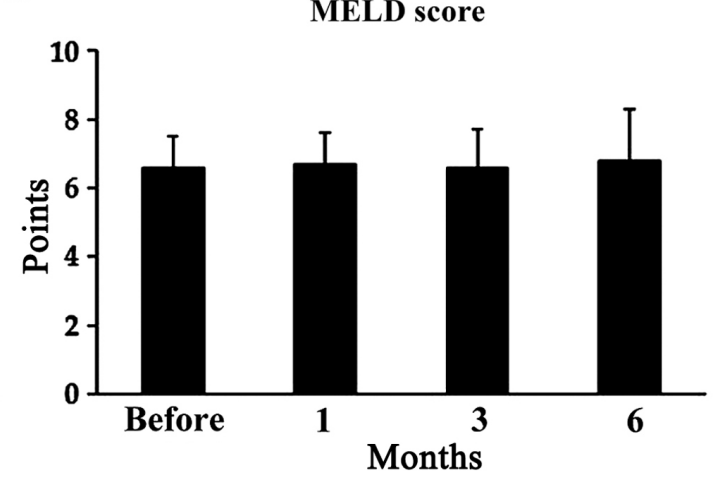

Figure 4. Changes in Child-Pugh and MELD scores during L-carnitine supplementation in patients with cirrhosis. L-carnitine supplementation did not have any significant effect on Child-Pugh or MELD scores. (A) Child-Pugh scores were 7.3 \pm 1.4 before treatment, 7.2 \pm 1.5 after 1 month, 7.2 \pm 1.6 after 3 months and $7.2 \pm 1.9$ after 6 months. (B) MELD scores were $6.6 \pm 0.9$ before treatment, $6.7 \pm 0.9$ after 1 month, $6.6 \pm 1.1$ after 3 months and $6.8 \pm 1.5$ after 6 months. MELD, Model For End-Stage Liver Disease.

A

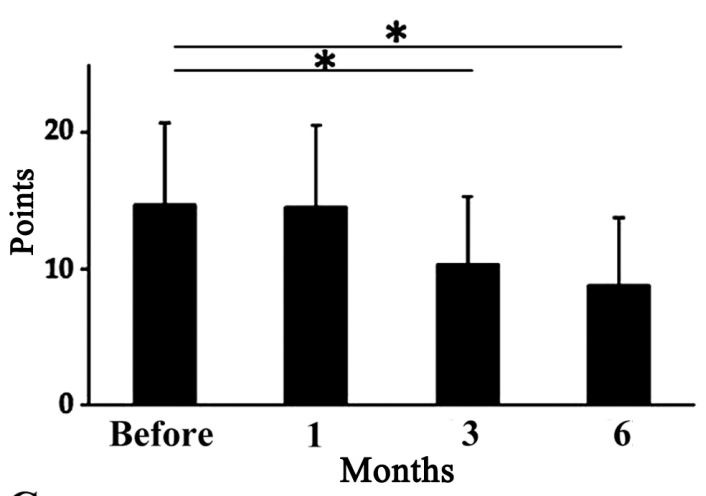

C

Mental score

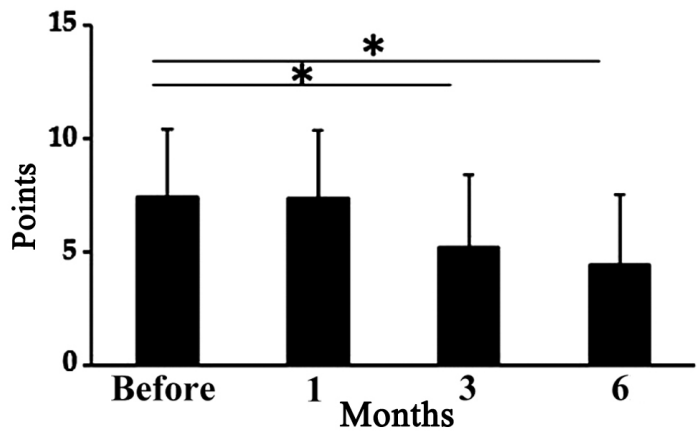

B

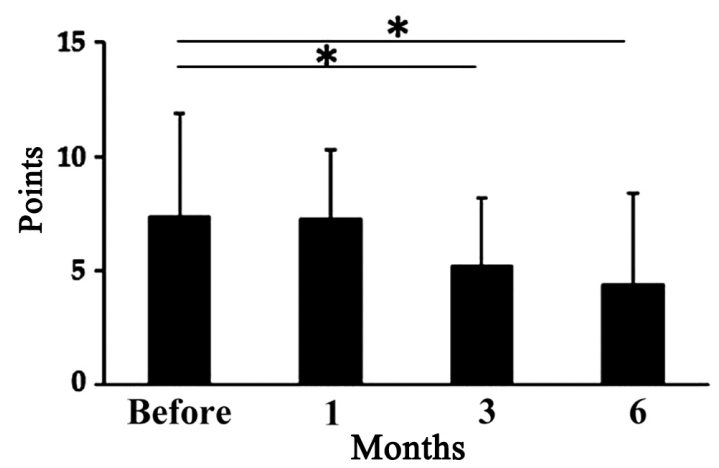

Figure 5. Changes in total scores, physical subscale scores and mental subscale scores on the Chronic Fatigue Questionnaire after L-carnitine supplementation was initiated in patients with cirrhosis. (A) Total scores were significantly lower after 3 months (10.3 \pm 5$)$ and 6 months $(8.74 \pm 5)$ of L-carnitine supplementation

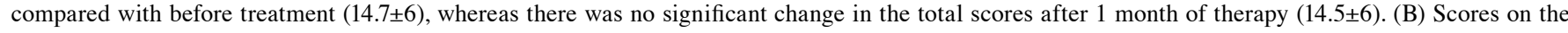
physical subscale showed a decreasing trend 3 and 6 months after therapy initiation: $7.37 \pm 4.5$ before treatment, $7.27 \pm 3.0$ after 1 month, $5.17 \pm 3.0$ after 3 months $(\mathrm{P}=0.057)$ and $4.37 \pm 4.0$ after 6 months $(\mathrm{P}=0.07)$. (C) Scores on the mental subscale were significantly lower after 3 and 6 months of therapy: 7.42 \pm 3.0 before treatment, $7.37 \pm 3.0$ after 1 month, $5.22 \pm 3.2$ after 3 months and $4.43 \pm 3.1$ after 6 months. ${ }^{*} \mathrm{P}<0.05$.

Effect of L-carnitine supplementation on chronic fatigue. The total scores on the Chronic Fatigue Questionnaire before treatment and after 1 month of L-carnitine supplementation did not differ significantly, but were significantly decreased after 3 and 6 months. The mean total scores were $14.7 \pm 6.0$ before treatment, $14.5 \pm 6.0$ after 1 month $(\mathrm{P}>0.05), 10.3 \pm 5.0$ points after 3 months $(\mathrm{P}<0.05)$ and $8.74 \pm 5.0$ after 6 months
$(\mathrm{P}<0.05$; Fig. 5A). On the physical subscale, scores were significantly decreased 3 and 6 months after therapy initiation; mean scores were $7.37 \pm 4.5$ points before treatment, $7.27 \pm 3.0$ points after 1 month $(\mathrm{P}>0.05), 5.17 \pm 3.0$ after 3 months $(\mathrm{P}<0.05)$ and $4.37 \pm 4.0$ after 6 months $(\mathrm{P}<0.05$; Fig. 5B). On the mental subscale, the scores were significantly decreased 3 and 6 months after therapy initiation; mean scores were 7.42 \pm 3.0 
A

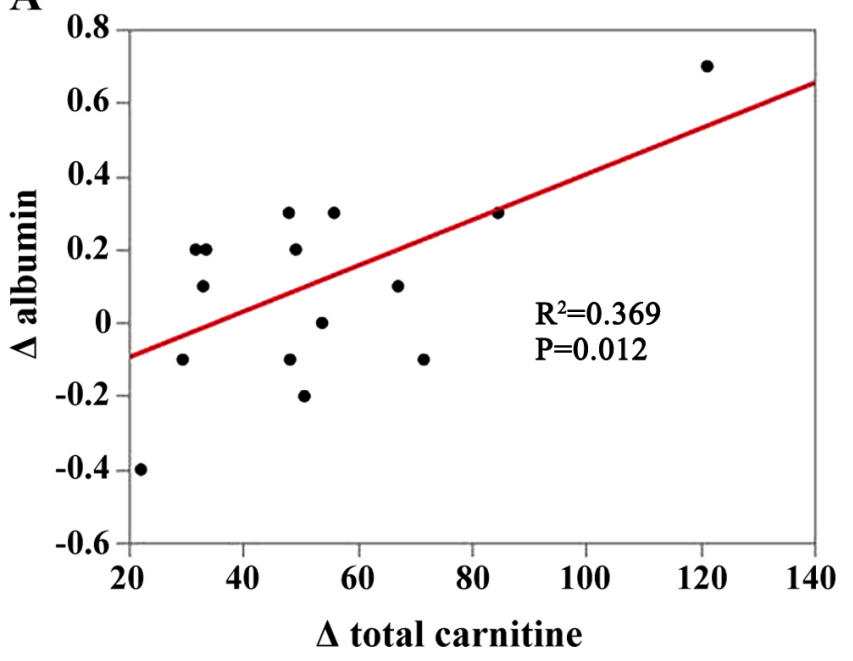

B

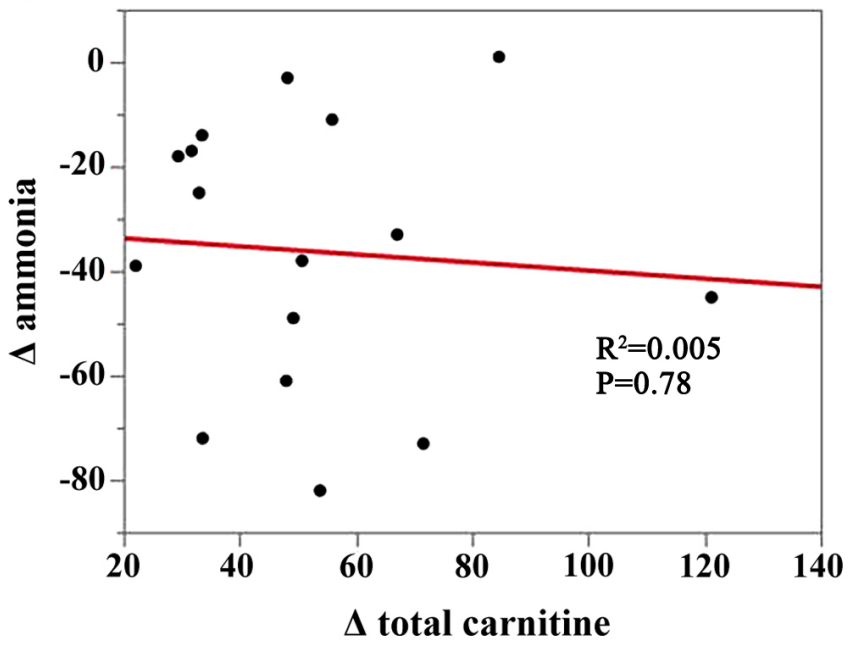

Figure 6. Correlation of changes in total carnitine levels with changes in serum albumin levels and serum ammonia levels. (A) Changes in serum carnitine concentrations were positively correlated with changes in serum albumin levels; (B) but not with changes in serum ammonia levels.

before treatment, $7.37 \pm 3.0$ points after 1 month $(\mathrm{P}>0.05)$ $5.22 \pm 3.2$ after 3 months $(\mathrm{P}<0.05)$ and $4.43 \pm 3.1$ after 6 months $(\mathrm{P}<0.05$; Fig. 5C).

Correlation of changes in total carnitine levels with changes in serum albumin levels and changes in serum ammonia levels. Changes in serum carnitine concentrations were positively correlated with changes in serum albumin levels $\left(\mathrm{R}^{2}=0.369 ; \mathrm{P}=0.012\right)$, but not with changes in serum ammonia levels $\left(\mathrm{R}^{2}=0.005 ; \mathrm{P}=0.78\right.$; Fig. $6 \mathrm{~A}$ and $\left.\mathrm{B}\right)$.

Effects of 6-month L-carnitine supplementation on the $d-R O M s / B A P$ ratio. L-carnitine supplementation did not significantly affect the serum d-ROM levels after 1 month; however, serum d-ROM levels were significantly lower 3 and 6 months after therapy initiation compared with before the treatment. Mean levels were 246.1 \pm 46.2 U.Carr before treatment, 225.2 \pm 50.6 U.Carr after 1 month $(P=0.67), 210.9 \pm 20.3$ U.Carr after 3 months $(\mathrm{P}<0.05)$ and 205.8 \pm 30.5 U.Carr after 6 months $(\mathrm{P}<0.05$; Fig. 7A). However, L-carnitine supplementation did not have any significant effect on serum BAP levels. Mean levels were 2,100 $\pm 322 \mu \mathrm{mol} / 1$ before treatment, $2,095 \pm 310 \mu \mathrm{mol} / 1$ after 1 month $(\mathrm{P}=0.52), 2,152 \pm 390 \mu \mathrm{mol} / 1$ after 3 months $(\mathrm{P}=0.43)$ and 2,120 $\pm 385 \mu \mathrm{mol} / \mathrm{l}$ after 6 months $(\mathrm{P}=0.28$; Fig. 7B). There was no significant effect of $\mathrm{L}$-carnitine supplementation after 1 month on the d-ROMs/BAP ratio, but the $\mathrm{d}-\mathrm{ROMs} / \mathrm{BAP}$ ratio was significantly higher 3 and 6 months after therapy initiation compared with before treatment initiation. The ratio of $\mathrm{d}-\mathrm{ROMs} / \mathrm{BAP}$ was $8.5 \pm 2.0$ before treatment, $9.3 \pm 2.2$ after 1 month $(\mathrm{P}=0.21), 10.2 \pm 1.9 \mu \mathrm{g} / \mathrm{dl}$ after 3 months $(\mathrm{P}<0.05)$ and $10.3 \pm 1.5$ after 6 months $(\mathrm{P}<0.05$; Fig. $7 \mathrm{C})$.

\section{Discussion}

L-carnitine is a nutrient prerequisite for energy generation in vital organs, including the liver, kidneys and brain $(29,30)$. Carnitine deficiency occurs in various disorders, such as malnutrition, liver cirrhosis, endocrine disorders and end-stage renal disease treated with hemodialysis, all of which are considered to affect health-related quality of life $(31,32)$. To the best of our knowledge, the present study is the first prospective study to show that $1,800 \mathrm{mg} /$ day L-carnitine supplementation for 6 months exhibited favorable effects on chronic fatigue in patients with cirrhosis. L-carnitine increased carnitine profiles, serum albumin levels, the ratio of BAP/d-ROM and decreased serum ammonia levels. L-carnitine increases carnitine palmitoyl transferase activity, which reflects mitochondrial fatty acid oxidation resulting in increased acylcarnitine levels (33). As changes in serum carnitine concentrations were positively correlated with changes in serum albumin levels, but not with changes in serum ammonia levels, this suggests that L-carnitine ameliorated chronic fatigue by attenuating oxidative stress.

A meta-analysis revealed that supplemental intake of 4,000 mg L-carnitine increases serum albumin levels and ameliorates serum ammonia levels in patients with hepatic encephalopathy (14). L-carnitine supplementation reduced the levels of ammonia in patients with cirrhosis with physiological blood carnitine levels (34), suggesting that increased quantities of carnitine is required for patients with cirrhosis compared with the healthy individuals, and that patients may have exhibited a relative carnitine deficiency. In contrast, $600 \mathrm{mg}$ oral L-carnitine daily was reported to counteract the reduction in serum albumin levels 1 week after transarterial chemoembolization (TACE) in patients with cirrhosis (35). These findings suggest that higher doses of L-carnitine supplementation may not be necessary for producing the therapeutic benefits as the homeostatic control of serum L-carnitine levels is tight and subtle (15). In the present study, it was shown that both Child-Pugh and MELD scores did not change significantly during the 6-month follow-up. However, L-carnitine supplementation maintains the levels of branched-chain amino acids for albumin synthesis, and this has been shown to improve Child-Pugh scores following TACE (35). The discrepancy may be partly explained by the differences in patient characteristics between the two studies. A systematic review and meta-analysis revealed beneficial effects of L-carnitine on serum albumin levels in patients with 
A

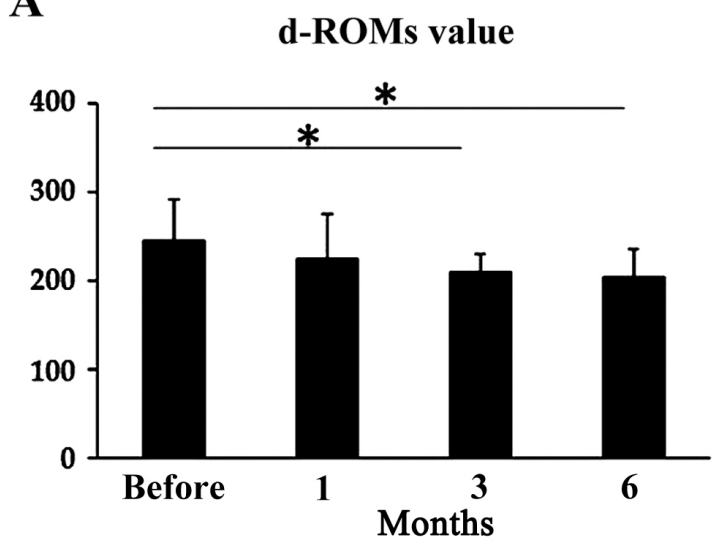

C

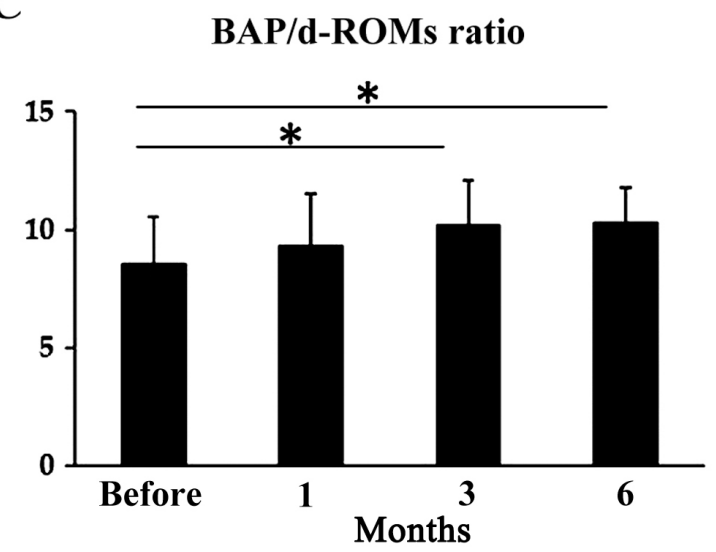

B

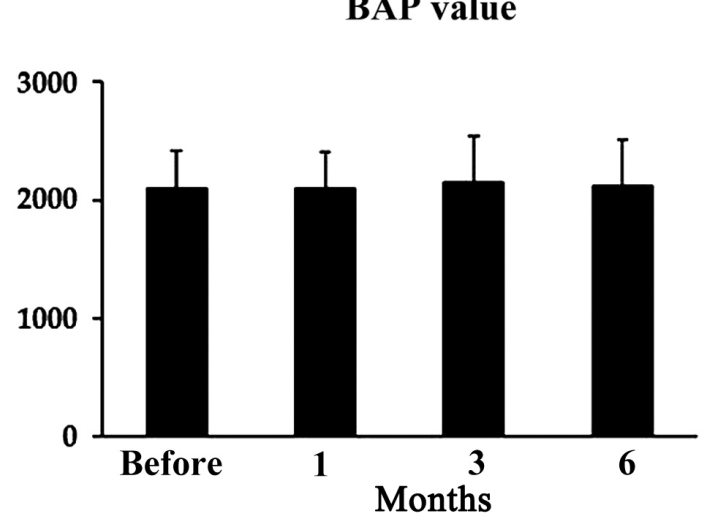

Figure 7. Changes in d-ROM levels, BAP levels and the BAP/d-ROMs ratio during L-carnitine supplementation in patients with cirrhosis. (A) Serum d-ROM levels were significantly lower after 3 months (210.9 \pm 20.3 U.Carr) and 6 months (205.8 \pm 30.5 U.Carr) of L-carnitine supplementation compared with before treatment (225.2 \pm 50.6 U.Carr). In contrast, there was no significant effect of L-carnitine supplementation for 1 month on serum albumin levels $(246.1 \pm 46.2$ U.Carr). (B) L-carnitine supplementation did not have any significant effect on serum BAP levels: $2,100 \pm 322 \mu \mathrm{mol} / 1$ before treatment and $2,095 \pm 310 \mu \mathrm{mol} / 1$ after 1 month, 2,152 $\pm 390 \mu \mathrm{mol} / 1$ after 3 months and 2,120 $\pm 385 \mu \mathrm{mol} / 1$ after 6 months. (C) The d-ROMs/BAP ratio was significantly higher after 3 and 6 months of therapy: $8.5 \pm 2.0$ before treatment, $9.3 \pm 2.2$ after 1 month, $10.2 \pm 1.9$ after 3 months and $10.3 \pm 1.5$ after 6 months. ${ }^{*}<<0.05$. d-ROM, d-reactive oxygen metabolite; BAP, biological antioxidant potential.

cirrhosis (14). However, L-carnitine did not increase the serum albumin levels in patients with nonalcoholic steatohepatitis, whose serum albumin levels were already relatively high (36). These data reinforce the fact that L-carnitine improves serum albumin levels in patients with cirrhosis, which is frequently complicated by hypoalbuminemia (37).

The complication of minimal encephalopathy may cause mental disorders in patients with cirrhosis. Administration of acetyl-L-carnitine, an acetylated form of L-carnitine improved the mental status to normal levels and resolved hyperammonemia in a patient on chronic hemodialysis who exhibited elevated serum ammonia levels and altered mental status (38). With acetyl-L-carnitine, a significant decrease in serum ammonia levels was observed in patients with cirrhosis, along with a significant improvement in mental function (39). Patients with hepatic encephalopathy treated with L-carnitine showed significant improvements with regard to mental fatigue (40). An intensity-dependent relationship was found between ammonia levels and exercise, which suggested that ammonia accumulation exhibits a pivotal role in both physical and mental fatigue (41). Malaguarnera et al (42) demonstrated that in patients with minimal hepatic encephalopathy, acetyl-L-carnitine improved the quality of life by reducing serum albumin levels. However, L-carnitine did not exert considerable effects on ammonia levels 3 months after L-carnitine supplementation (42). The causes of differences in serum carnitine profiles between the studies are unclear. However, one possible explanation is the differences in the causes of liver cirrhosis and in the functional reserve capacities in patients $(43,44)$. Furthermore, in the present study, changes in total carnitine levels were not significantly correlated with changes in ammonia levels. These findings suggest that the favorable effects of L-carnitine on chronic fatigue in patients with cirrhosis cannot be attributed to improvements in serum ammonia levels.

The mechanism underlying the favorable effects of L-carnitine on chronic fatigue remain unclear. L-carnitine supplementation increases albumin levels in patients with cirrhosis. Patients receiving L-carnitine treatment showed improvements in daily activity performance levels and the performance efficiency of high-intensity muscular exercise, through the regulation of mitochondrial homeostasis (45). More recent evidence has suggested a close association between chronic fatigue and oxidative stress (46). In the present study, it was shown that L-carnitine supplementation increased the BAP/d-ROM ratio with serum albumin levels in patients with cirrhosis. Albumin is involved in several bioactive functions, such as antioxidant activity (47). 
Furthermore, serum albumin reportedly decreased oxidative stress by inhibiting the activation of nicotinamide adenine dinucleotide phosphate oxidase in human vascular smooth muscle (48). In addition, L-carnitine supplementation augmented fat oxidation and energy expenditure during prolonged exercise, which therefore spares glycogen stores and delays the onset of fatigue (39). These findings suggest that L-carnitine supplementation can potentially ameliorate chronic fatigue by attenuating oxidative stress (49). However, the exact mechanisms by which L-carnitine ameliorates chronic fatigue requires further investigation.

Several limitations associated with the present study must be mentioned. First, it was not possible to compare patients who received L-carnitine treatment with patients who did not. Second, the relationship between physical function and skeletal muscle mass were not assessed, which may explain the effect of L-carnitine on sarcopenia. Furthermore, the sample size was relatively small. A future study with a larger cohort is required to confirm the results of the present study. However, the present study is the first prospective clinical trial in which the beneficial effects of L-carnitine supplementation on physical and mental functional abilities in patients with cirrhosis and chronic fatigue were assessed through the use of a self-report questionnaire. The results showed that improvement in carnitine profiles were accompanied by increases in serum albumin levels, but not changes in serum ammonia levels. This suggests that L-carnitine ameliorates chronic fatigue by attenuating oxidative stress. L-carnitine alleviated chronic fatigue and increased serum albumin levels, which are recognized markers of antioxidant status in patients with cirrhosis. Nevertheless, larger studies are required to evaluate the effects of carnitine on health-related quality of life in patients with cirrhosis and investigate the exact mechanisms by which L-carnitine ameliorates chronic fatigue.

\section{Acknowledgements}

The authors would like to thank Ms Nakai (Nara Medical University) for assistance in collecting the clinical data.

\section{Funding}

This study was partially supported by Grants-in-Aid for Scientific Research from Nara Medical University (grant no. $15 \mathrm{~K} 08077)$.

\section{Availability of data and materials}

The datasets used and/or analyzed during the present study are available from the corresponding author on reasonable request.

\section{Authors' contributions}

SSat, MF, SSai, HK, KKa, HT, NS, YS, KKi, KM, TAk, NH and TAn performed the data analysis. AM, HY and TN made substantial contributions to the conception and design of the study, as well as analysis and interpretation of the data. All authors read and approved the final manuscript.

\section{Ethics approval and consent to participate}

Informed consent was obtained from all participants before the initiation of the study. This study was approved by the Human Ethics Review Committee of Bellland General Hospital (Osaka, Japan), (approval no. 2015-0004) and was performed in accordance with the Declaration of Helsinki.

\section{Patient consent for publication}

Not applicable.

\section{Competing interests}

The authors declare that they have no competing interests.

\section{References}

1. Oney-Birol S: Exogenous L-carnitine promotes plant growth and cell division by mitigating genotoxic damage of salt stress. Sci Rep 9: 17229, 2019.

2. Rinaudo MT, Curto M, Bruno R, Piccinini M and Marino C: Acid soluble, short chain esterified and free carnitine in the liver, heart, muscle and brain of pre and post hatched chicks. Int J Biochem 23: 59-65, 1991.

3. Khan L and Bamji MS: Tissue carnitine deficiency due to dietary lysine dificiency: Triglyceride accumulation and concomitant impairment in fatty acid oxidation. J Nutr 109: 24-31, 1979.

4. Rebouche CJ: Carnitine function and requirements during the life cycle. FASEB J 6: 3379-3386, 1992.

5. Cimmino A, Andolfi A, Zonno MC, Troise C, Santini A, Tuzi A, Vurro M, Ash G and Evidente A: Phomentrioloxin: A phytotoxic pentasubstituted geranylcyclohexentriol produced by Phomopsis sp., a potential mycoherbicide for Carthamus lanatus Biocontrol. J Nat Prod 75: 1130-1137, 2012.

6. Longo N, Frigeni M and Pasquali M: Carnitine transport and fatty acid oxidation. Biochim Biophys Acta 1863: 2422-2435, 2016.

7. Ringseis R, Keller $\mathrm{J}$ and Eder $\mathrm{K}$ : Role of carnitine in the regulation of glucose homeostasis and insulin sensitivity: Evidence from in vivo and in vitro studies with carnitine supplementation and carnitine deficiency. Eur J Nutr 51: 1-18, 2012.

8. Irvine KM, Ratnasekera I, Powell EE and Hume DA: Causes and consequences of innate immune dysfunction in cirrhosis. Front Immunol 10: 293, 2019.

9. Malaguarnera M, Vacante M, Motta M, Giordano M, Malaguarnera G, Bella R, Nunnari G, Rampello L and Pennisi G: Acetyl-L-carnitine improves cognitive functions in severe hepatic encephalopathy: A randomized and controlled clinical trial. Metab Brain Dis 26: 281-289, 2011.

10. Cecere A, Ciaramella F, Tancredi L, Romano C and Gattoni A: Efficacy of L-carnitine in reducing hyperammonaemia and improving neuropsychological test performance in patients with hepatic cirrhosis: Results of a randomised trial. Clin Drug Investig 22 (Suppl 1): S7-S14, 2002.

11. Hiramatsu A, Aikata H, Uchikawa S, Ohya K, Kodama K, Nishida Y, Daijo K, Osawa M, Teraoka Y, Honda F, et al: Levocarnitine use is associated with improvement in sarcopenia in patients with liver cirrhosis. Hepatol Commun 3: 348-355, 2019.

12. Nakanishi H, Kurosaki M, Tsuchiya K, Nakakuki N, Takada H, Matsuda S, Gondo K, Asano Y, Hattori N, Tamaki N, et al: L-carnitine reduces muscle cramps in patients with cirrhosis. Clin Gastroenterol Hepatol 13: 1540-1543, 2015.

13. Tajiri K, Futsukaichi Y, Kobayashi S, Yasumura S, Takahara T, Minemura M and Sugiyama T: L-carnitine for the treatment of overt hepatic encephalopathy in patients with advanced liver cirrhosis. J Nutr Sci Vitaminol (Tokyo) 64: 321-328, 2018.

14. Abbasnezhad A, Choghakhori R, Kashkooli S, Alipour M, Asbaghi $\mathrm{O}$ and Mohammadi R: Effect of L-carnitine on liver enzymes and biochemical factors in hepatic encephalopathy: A systematic review and meta-analysis. J Gastroenterol Hepatol 34: 2062-2070, 2019. 
15. Shang R, Sun $\mathrm{Z}$ and Li H: Effective dosing of L-carnitine in the secondary prevention of cardiovascular disease: A systematic review and meta-analysis. BMC Cardiovasc Disord 14: 88, 2014.

16. Howanitz JH, Howanitz PJ, Skrodzki CA and Iwanski JA Influences of specimen processing and storage conditions on results for plasma ammonia. Clin Chem 30: 906-908, 1984.

17. Bajaj JS, Cordoba J, Mullen KD, Amodio P, Shawcross DL, Butterworth RF and Morgan MY; International Society for Hepatic Encephalopathy and Nitrogen Metabolism (ISHEN): Review article: The design of clinical trials in hepatic encephalopathy-an International Society for Hepatic Encephalopathy and Nitrogen Metabolism (ISHEN) consensus statement. Aliment Pharmacol Ther 33: 739-747, 2011.

18. Michalak A, Rose C, Butterworth $\mathrm{J}$ and Butterworth RF Neuroactive amino acids and glutamate (NMDA) receptors in frontal cortex of rats with experimental acute liver failure. Hepatology 24: 908-913, 1996.

19. Peng Y, Qi X and Guo X: Child-pugh versus MELD score for the assessment of prognosis in liver cirrhosis: A systematic review and meta-analysis of observational studies. Medicine (Baltimore) 95: e2877, 2016.

20. Tanaka S, Kuratsune H, Hidaka Y, Hakariya Y, Tatsumi KI, Takano T, Kanakura Y and Amino N: Autoantibodies against muscarinic cholinergic receptor in chronic fatigue syndrome. Int J Mol Med 12: 225-230, 2003.

21. Okada T, Tanaka M, Kuratsune H, Watanabe Y and Sadato N: Mechanisms underlying fatigue: A voxel-based morphometric study of chronic fatigue syndrome. BMC Neurol 4: 14, 2004.

22. Matsuda Y, Matsui T, Kataoka K, Fukada R, Fukuda S, Kuratsune H, Tajima S, Yamaguti K, Kato YH and Kiriike N: A two-year follow-up study of chronic fatigue syndrome comorbid with psychiatric disorders. Psychiatry Clin Neurosci 63: 365-373, 2009.

23. General Assembly of the World Medical Association: World Medical Association Declaration of Helsinki: Ethical principles for medical research involving human subjects. J Am Coll Dent 81: 14-18, 2014.

24. Morimoto M, Hashimoto T, Tsuda Y, Kitaoka T and Kyotani S Evaluation of oxidative stress and antioxidant capacity in healthy children. J Chin Med Assoc 82: 651-654, 2019.

25. Celi P, Sullivan M and Evans D: The stability of the reactive oxygen metabolites (d-ROMs) and biological antioxidant potential (BAP) tests on stored horse blood. Vet J 183: 217-218, 2010.

26. Bergami R, Maranesi M, Marchetti M, Sangiorgi Z and Tolomelli B: Influence of dietary n-3 polyunsaturated fatty acids on plasma lipemic effect of vitamin B6 deficiency. Int J Vitam Nutr Res 69: 315-321, 1999.

27. Kuratsune H, Yamaguti K, Lindh G, Evengård B, Hagberg G Matsumura K, Iwase M, Onoe H, Takahashi M, Machii T, Kanakura Y, et al: Brain regions involved in fatigue sensation: Reduced acetylcarnitine uptake into the brain. Neuroimage 17: 1256-1265, 2002.

28. Mizuno K, Tanaka M, Nozaki S, Mizuma H, Ataka S, Tahara T, Sugino T, Shirai T, Kajimoto Y, Kuratsune H, Kajimoto O and Watanabe Y: Antifatigue effects of coenzyme Q10 during physical fatigue. Nutrition 24: 293-299, 2008.

29. Malaguarnera M: Carnitine derivatives: Clinical usefulness. Curr Opin Gastroenterol 28: 166-176, 2012.

30. Kendler BS: Carnitine: An overview of its role in preventive medicine. Prev Med 15: 373-390, 1986.

31. Malaguarnera M: Acetyl-L-carnitine in hepatic encephalopathy. Metab Brain Dis 28: 193-199, 2013.

32. Gonwa TA and Wadei HM: Kidney disease in the setting of liver failure: Core curriculum. Am J Kidney Dis 2013 62: 1198-1212, 2013.
33. Flanagan JL, Simmons PA, Vehige J, Willcox MD and Garrett Q: Role of carnitine in disease. Nutr Metab (Lond) 7: 30, 2010.

34. Shiraki M, Shimizu M, Moriwaki H, Okita K and Koike K Carnitine dynamics and their effects on hyperammonemia in cirrhotic Japanese patients. Hepatol Res 47: 321-327, 2017.

35. Hassan A, Tsuda Y, Asai A, Yokohama K, Nakamura K, Sujishi T, Ohama H, Tsuchimoto Y, Fukunishi S, Abdelaal UM, Arafa UA, et al: Effects of oral L-carnitine on liver functions after transarterial chemoembolization in intermediate-stage HCC patients. Mediators Inflamm 2015: 608216, 2015.

36. Malaguarnera M, Gargante MP, Russo C, Antic T, Vacante M, Malaguarnera M, Avitabile T, Li Volti G and Galvano F: L-carnitine supplementation to diet: A new tool in treatment of nonalcoholic steatohepatitis-a randomized and controlled clinical trial. Am J Gastroenterol 105: 1338-1345, 2010.

37. Soeters PB, Wolfe RR and Shenkin A: Hypoalbuminemia: Pathogenesis and clinical significance. JPEN J Parenter Enteral Nutr 43: 181-193, 2019.

38. DaVanzo WJ and Ullian ME: L-carnitine administration reverses acute mental status changes in a chronic hemodialysis patient with hepatitis C infection. Clin Nephrol 57: 402-405, 2002.

39. Stephens FB, Constantin-Teodosiu D and Greenhaff PL: New insights concerning the role of carnitine in the regulation of fuel metabolism in skeletal muscle. J Physiol 581: 431-444, 2007.

40. Malaguarnera M, Gargante MP, Cristaldi E, Vacante M, Risino C, Cammalleri L, Pennisi G and Rampello L: Acetyl-L-carnitine treatment in minimal hepatic encephalopathy. Dig Dis Sci 53 3018-3025, 2008

41. Malaguarnera M, Vacante M, Giordano M, Pennisi G, Bella R, Rampello L, Malaguarnera M, Li Volti G and Galvano F: Oral acetyl-L-carnitine therapy reduces fatigue in overt hepatic encephalopathy: A randomized, double-blind, placebo-controlled study. Am J Clin Nutr 93: 799-808, 2011.

42. Malaguarnera M,Bella R, Vacante M, Giordano M, Malaguarnera G, Gargante MP, Motta M, Mistretta A, Rampello L and Pennisi G: Acetyl-L-carnitine reduces depression and improves quality of life in patients with minimal hepatic encephalopathy. Scand J Gastroenterol 46: 750-759, 2011.

43. Amodio P, Angeli P, Merkel C, Menon F and Gatta A: Plasma carnitine levels in liver cirrhosis: Relationship with nutritional status and liver damage. J Clin Chem Clin Biochem 28: 619-626, 1990.

44. D'Arienzo A, Mattera D, Ambrogio G, Duranti L, Zeuli L and Mazzacca G: Hypercarnitinemia in cirrhosis. Hepatology 5: 343, 1985.

45. Gimenes AC, Bravo DM, Napolis LM, Mello MT, Oliveira AS, Neder JA and Nery LE: Effect of L-carnitine on exercise performance in patients with mitochondrial myopathy. Braz J Med Biol Res 48: 354-362, 2015.

46. Lee JS, Kim HG, Lee DS and Son CG: Oxidative stress is a convincing contributor to idiopathic chronic fatigue. Sci Rep 8: 12890, 2018

47. Sitar ME, Aydin S and Cakatay U: Human serum albumin and its relation with oxidative stress. Clin Lab 59: 945-952, 2013.

48. Kinoshita H, Watanabe K, Azma T, Feng GG, Akahori T, Hayashi H, Sato M, Fujiwara Y and Wakatsuki A: Human serum albumin and oxidative stress in preeclamptic women and the mechanism of albumin for stress reduction. Heliyon 3: e00369, 2017.

49. Malaguarnera M, Pistone G, Astuto M, Vecchio I, Raffaele R, Lo Giudice E and Rampello L: Effects of L-acetylcarnitine on cirrhotic patients with hepatic coma: Randomized double-blind, placebo-controlled trial. Dig Dis Sci 51: 2242-2247, 2006.

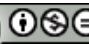

This work is licensed under a Creative Commons Attribution-NonCommercial-NoDerivatives 4.0 International (CC BY-NC-ND 4.0) License. 